\title{
Monitoring the atmospheric turbulence profile with high vertical resolution with the PML instrument
}

\author{
Julien Chabé ${ }^{a}$, Eric Aristidi ${ }^{b}$, Aziz Ziad $^{b}$, Yan Fantei-Caujolle ${ }^{b}$, Henri Lantéri ${ }^{b}$ Christophe $^{b}$ \\ Giordano $^{b}$, Julien Borgnino ${ }^{b}$, Catherine Renaud ${ }^{b}$, \\ ${ }^{a}$ Université Côte d'Azur, Observatoire de la Côte d'Azur, CNRS, IRD, Géoazur, 2130 route de \\ l'Observatoire, 06460 Caussols, France \\ ${ }^{b}$ UMR 7293 Lagrange, Université de Nice-Sophia Antipolis, CNRS, OCA, Parc Valrose \\ F-06108 Nice Cedex 2, France
}

\begin{abstract}
Wide-Field Adaptive Optics systems will be a key component of the future generation of Extremely Large Telescopes. All the components of such Adaptive Optics systems have to be precisely specified using the atmospheric turbulence parameters of the site, particularly the profile of the refractive index structure constant $C_{n}^{2}(h)$. The monitor PML (Profiler of Moon Limb), for the extraction of the $C_{n}^{2}(h)$ profile with high vertical resolution for nighttime and daytime conditions, has been developed and is now routinely exploited at the Calern Observatory (French Riviera). The PML instrument uses a differential method with two small subapertures mask through which the Moon limb or Sun edge are observed leading to a continuum of double stars allowing a scan of the whole atmosphere with high resolution in altitude. In addition, PML provides, in real time, a complete characterization of the atmospheric turbulence since it is able to measure the other turbulence parameters like the total seeing and the isoplanatic angle.
\end{abstract}

Keywords: Site-testing, Adaptive Optics, Interferometry.

\section{INTRODUCTION}

Wide-Field Adaptive Optics (WFAO) have been proposed for the large field compensation in the perspective of the future Extremely Large Telescopes (ELTs). ${ }^{1-3}$ The optimization of the performances of the WFAO techniques requires a precise specification of the different components of these systems. Some of these technical specifications are related to the optical parameters of the atmospheric turbulence such as seeing and isoplanatic angle. Recent studies $^{4,5}$ show that it is necessary to have access to $C_{n}^{2}(h)$ profiles with high vertical resolution for a better evaluation of the performance of a WFAO system.

Precise nocturnal and diurnal knowledge of the $C_{n}^{2}(h)$ profiles (as well as wind and outer scale profiles) with high vertical resolution is also needed for Free Space Optics (FSO) for communications or for Time and Frequency Transfer in Two Way Coherent Optical Link ${ }^{6}$ between earth and artificial satellites. Wave optics simulations to evaluate the dramatic effect of scintillation of the optical signal on fibre injection and the estimated Bit Error Rate $(\mathrm{BER})^{7,8}$ must be feeded with those profiles. This helps for designing specific AO systems with higher bandwidth and capable of working over a high variability of atmospheric conditions. Real-time evaluation of accessibility of the atmospheric communication channel based on this kind of simulation will also be a need for future FSO networks. ${ }^{9}$

The optical turbulence forecasting, necessary for both astronomical observations scheduling ${ }^{10,11}$ and optical links, is done using a numerical weather system coupled with an optical turbulence model. The calibration of these two models is then required to reach the best possible precision. The best way to do, is to use local meteorological measurements to parametrize the weather model, and local profiles (daytime and night time) of $C_{n}^{2}(h)$ to constrain the optical turbulence model. Having a high vertical resolution $C_{n}^{2}(h)$ profiles is important to consider, at best, phenomena within the surface layer.

Further author information: (Send correspondence to J.C.): E-mail: julien.chabe@oca.eu, Telephone: (33) (0)4 93 40 5410 
Our instrument PML (Profiler of Moon Limb) has been developed for the extraction of the $C_{n}^{2}(h)$ profile with high vertical resolution from Moon limb fluctuations. The Moon or Sun limb acts as a continuum of double stars with all possible angular separations required between two points to scan the atmosphere with a very fine resolution (see Fig.1). The PML is the only instrument with the ability to acquire data during daylight conditions, this is an advantage for a better modeling of atmospheric turbulence. ${ }^{12}$
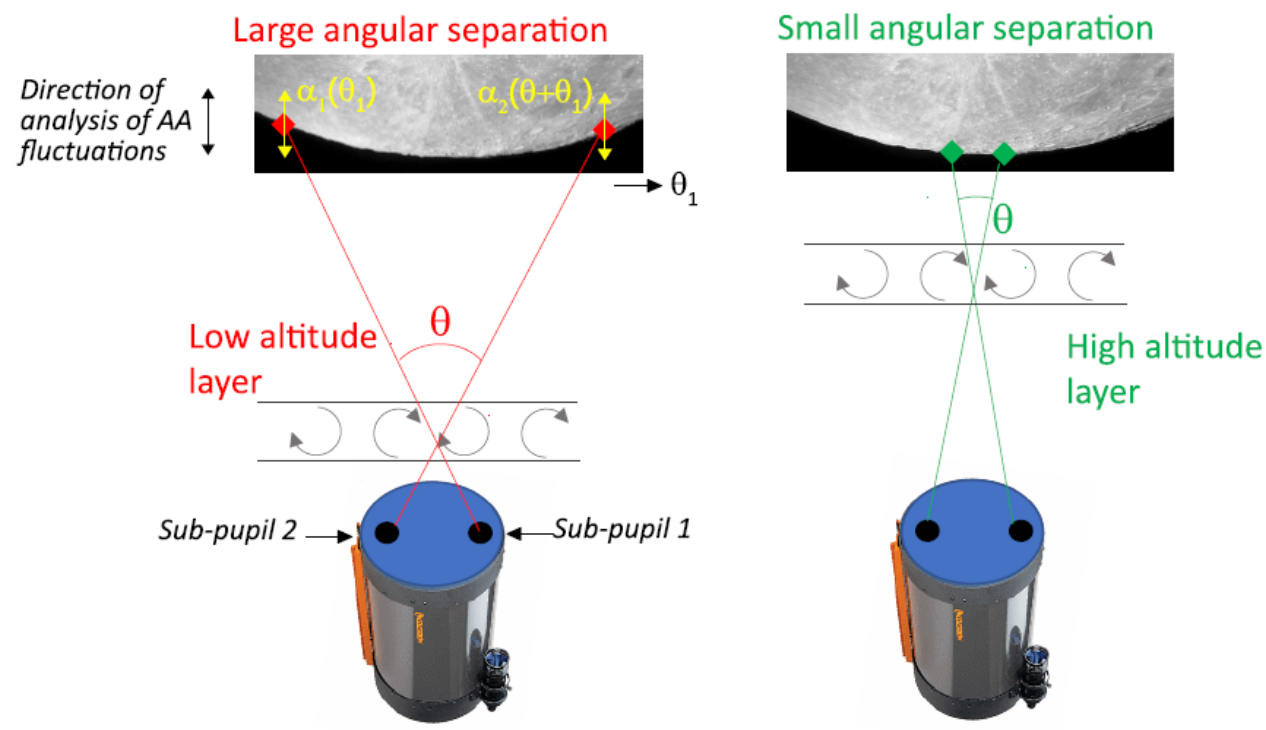

Figure 1. Principle of PML measurement of the covariance of AA differential motion between two points on the Moon limb. Large (resp. small) angular separations give access to low (resp. high) altitude layers.

\section{PRINCIPLE OF THE MEASUREMENT}

The PML instrument measures the wavefront angle-of-arrival (AA) from the motion of the Moon limb (or Sun edge) image. PML uses the differential method of the Differential Image Motion Monitor (DIMM) ${ }^{13}$ through two subapertures of diameter $D$ separated by a baseline $B$. Two images of the Moon or the Sun are produced at the focal plane of a $40 \mathrm{~cm}$ diameter telescope. A Dove prism is used to produce a mirror image of the Moon/Sun from one of the two subapertures. Fig.2 shows a photograph of the telescope in its dome at Calern Observatory. The pupil mask has two sub-apertures of diameter $D=6 \mathrm{~cm}$ separated by a baseline $B=27 \mathrm{~cm}$. The baseline is parallel to the declination axis of the equatorial mount. Fig.3 shows the optical scheme of the PML bench. The camera is a PCO Pixelfly CCD with a matrix of $640 \times 480$ pixels, working in the visible domain (the bandwidth FWHM is $320-630 \mathrm{~nm}$ ). The angular sampling of $0.57 \mathrm{arcsec} /$ pixel is a good compromise between the spatial resolution and the field of view $(365 \operatorname{arcsec})$. Every $\sim 4$ minutes a 1024 images cube with an exposure time of 5 milliseconds is acquired to compute a $C_{n}^{2}(h)$ profile.

\section{THEORETICAL BACKGROUND}

The theoretical background of PML is presented in details in. ${ }^{14}$ The observed Moon or Sun limb is parallel to the sub-apertures baseline and the instrument measures the differential AA through the two subapertures, the transverse covariance of the differential AA fluctuations $\Delta \alpha$ between the two images from the two subapertures is then computed:

$$
C_{\Delta \alpha}(\theta)=\left\langle\left[\Delta \alpha\left(r, \theta_{0}\right)\right]\left[\Delta \alpha\left(r, \theta_{0}+\theta\right)\right]\right\rangle
$$

where $\Delta \alpha\left(r, \theta_{0}\right)$ and $\Delta \alpha\left(r, \theta+\theta_{0}\right)$ represent the differential fluctuations of the lunar (or solar) limb image observed between subapertures of the PML and measured at the angular positions $\theta_{0}$ and $\theta+\theta_{0}$. This differential angular covariance is given by: ${ }^{15}$ 


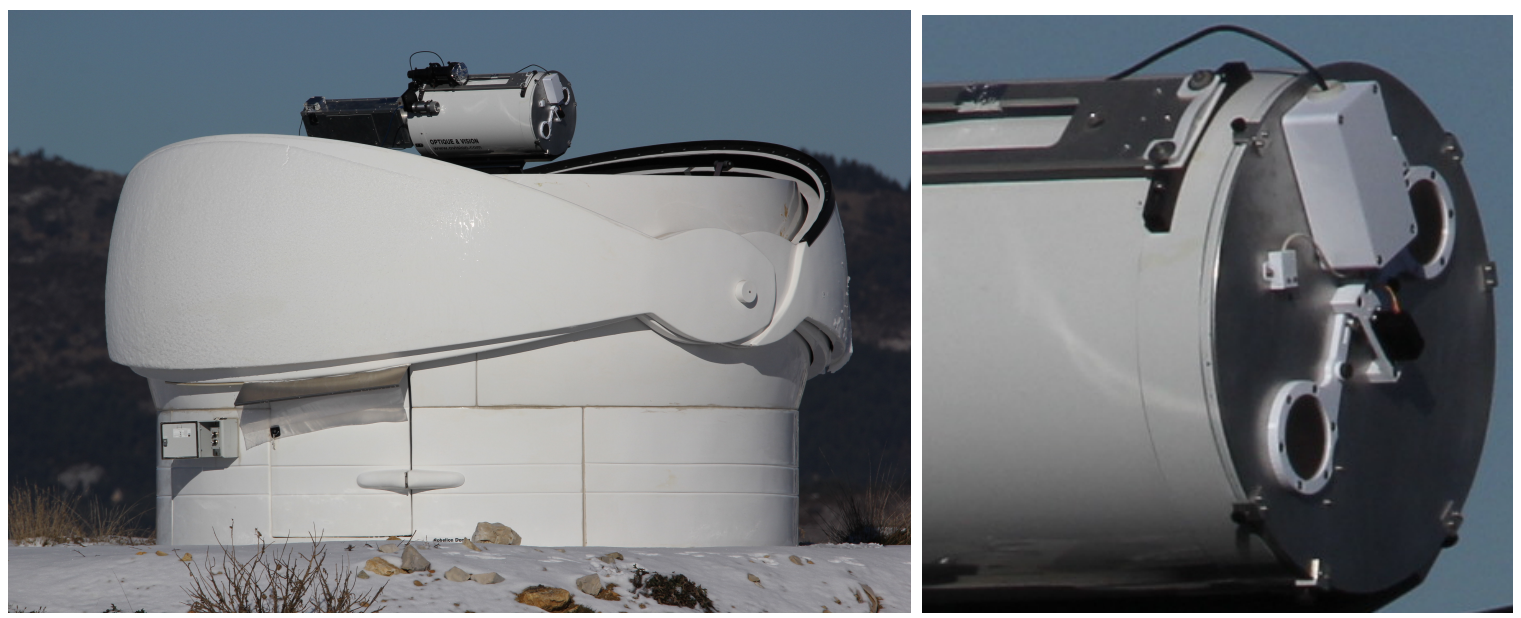

Figure 2. Left: the Profiler of Moon Limb instrument inside its 12ft AllSky dome. Right: The collapsible solar filters for Sun observation.
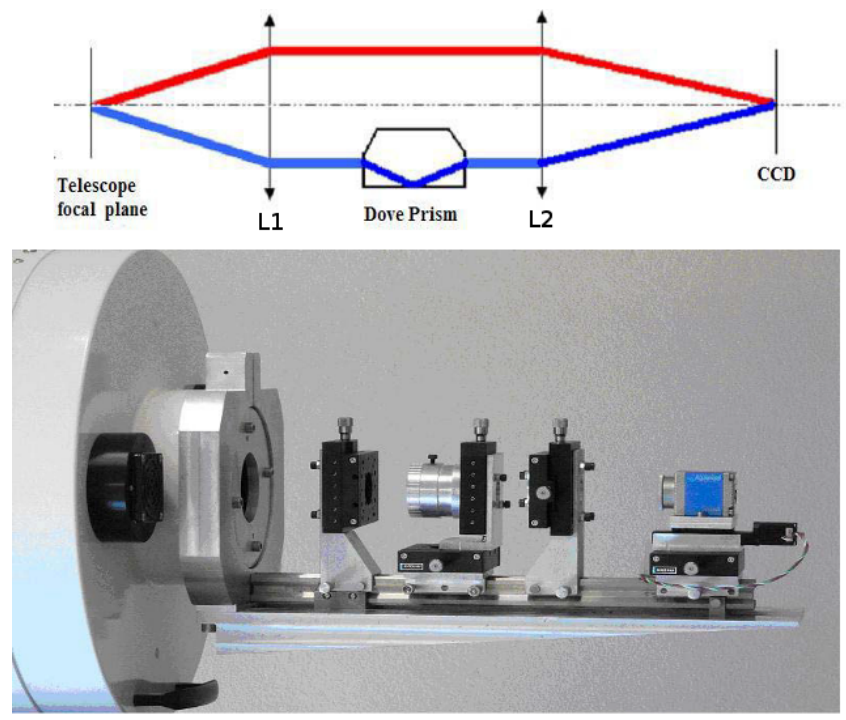

Figure 3. The optical device of the PML instrument.

$$
C_{\Delta \alpha}(\theta)=\int d h C_{n}^{2}(h) K_{\alpha}(B, h, \theta)
$$

where the kernel $K_{\alpha}(B, h, \theta)=2 C_{\alpha}(\theta h)-C_{\alpha}(B-\theta h)-C_{\alpha}(B+\theta h)$ is a triplet of normalized spatial covariances. In the case of the von Kàrmàn model for a baseline $\varrho$, a sub-aperture diameter $\mathrm{D}$ (here $6 \mathrm{~cm}$ ), and a single layer at altitude $h, C_{\alpha}(\varrho)$ is given in ${ }^{16}$ by:

$$
C_{\alpha}(\varrho)=1.19 \sec (z) \int d f f^{3}\left(f^{2}+\frac{1}{\mathcal{L}_{0}^{2}}\right)^{-11 / 6} \times\left[J_{0}(2 \pi \varrho f)+J_{2}(2 \pi \varrho f)\right]\left[2 \frac{J_{1}(\pi D f)}{\pi D f}\right]^{2},
$$

where $z$ is the zenithal angle, $f$ the spatial frequency, $D$ the sub-aperture diameter and $\mathcal{L}_{0}$ the outer scale.

We used the assumption of a discrete turbulent profile to transform eq. 2 into:

$$
C_{\Delta \alpha}(\theta)=\sum \Delta h_{i} C_{n}^{2}\left(h_{i}\right) \widehat{K}_{\alpha}\left(B, h_{i}, \theta\right)
$$


where $\widehat{K}_{\alpha}\left(B, h_{i}, \theta\right)$ is the modified spatial covariance triplet and $\Delta h_{i}$ is the thickness of the layer $i$. This equation takes a matrix form: $Y=M . X$ where $X$ and $Y$ are both vectors corresponding respectively to the sampled $C_{n}^{2}\left(h_{i}\right)$ and the covariance difference $C_{\Delta \alpha}(\theta)$, the matrix $M$ contain the modified spatial covariance triplet weighted with $\Delta h_{i}: M=\widehat{K}_{\alpha}\left(B, h_{i}, \theta\right) . \Delta h_{i}$. The values of $h_{i}$ ranges from 50 to $950 \mathrm{~m}$ with a thickness $\Delta h_{i}$ of $100 \mathrm{~m}$, from $1250 \mathrm{~m}$ to $4750 \mathrm{~m}$ with $\Delta h_{i}=500 \mathrm{~m}$, from $5500 \mathrm{~m}$ to $14500 \mathrm{~m} \Delta h_{i}=1000 \mathrm{~m}$ and from $16000 \mathrm{~m}$ to $24000 \mathrm{~m}$ with a thickness $\Delta h_{i}$ of $2000 \mathrm{~m}$. The value for the outer scale $\mathcal{L}_{0}$ in the kernel is taken equal to $20 \mathrm{~m}$. It has indeed very little influence on differential covariances. ${ }^{15}$ The $C_{n}^{2}(h)$ profile is retrieved by solving an inverse problem on eq.4 under non-negativity constraint, via the minimization of a least squares criterion between measured and modelled differential covariances.

\section{SITE TESTING AT THE CALERN OBSERVATORY WITH PML}

The PML is part of the Calern Atmospheric Turbulence Station (CATS) located at the Calern plateau (1261m, $43.75^{\circ} \mathrm{N}, 6.88^{\circ} \mathrm{E}$ ). The CATS station also include a Differential Image Motion Moniteur (G-DIMM) and has been designed to be autonomous and it is equipped with an allsky camera to detect the presence of clouds in the sky and a weather station for monitoring, temperature, humidity, dew point and wind velocity. All these sensors information is treated by a software devoted to observation management. While observing the PML sends its data to a website available for all the users of the observatory. The webpage shows, as a function of time, meteorological data, a picture of the night sky, the fraction of sky covered with clouds, the seeing, the scintillation and the isoplanetism angle and the $C_{n}^{2}(h)$ profile.

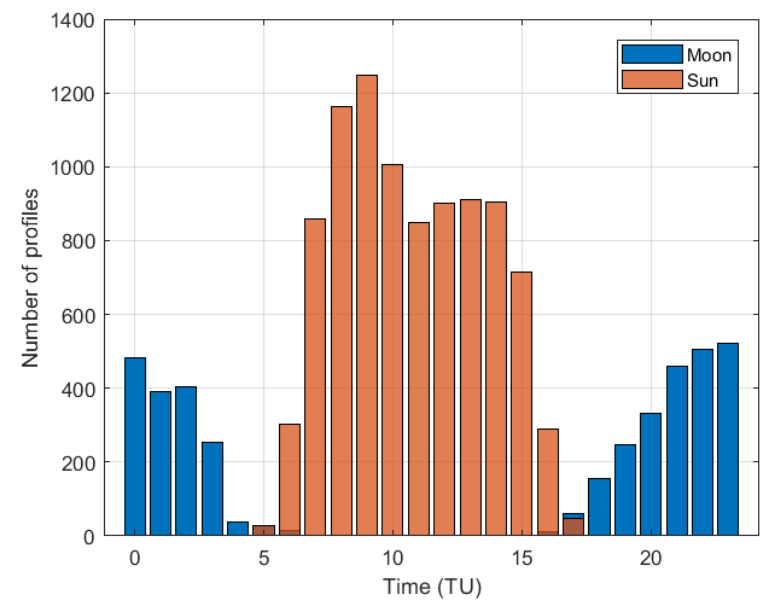

Figure 4. Number of $C_{n}^{2}(h)$ profiles acquired per hour over the year 2019

Over the year 2019, a total of 13126 profiles have been acquired by the PML instrument: 9224 on the Sun (during daytime) and 3902 on the Moon (nighttime). The data set is larger on the Sun, since the Moon is not always present in the sky (especially in Summer where the Moon has a low altitude above the horizon close to the full Moon). Fig.4 shows the histogram of the number of $C_{n}^{2}(h)$ profiles over 24 hours for the year 2019. Using the both target, Sun and Moon, the PML is able to cover almost an entire day with however a lack of data around sunrise and sunset. Fig.5 represents the number of $C_{n}^{2}(h)$ profiles per day for the Moon and the Sun over the year 2019. The instrument reaches a median value of 41 profiles per day for the Moon and 73 profiles for the Sun. 


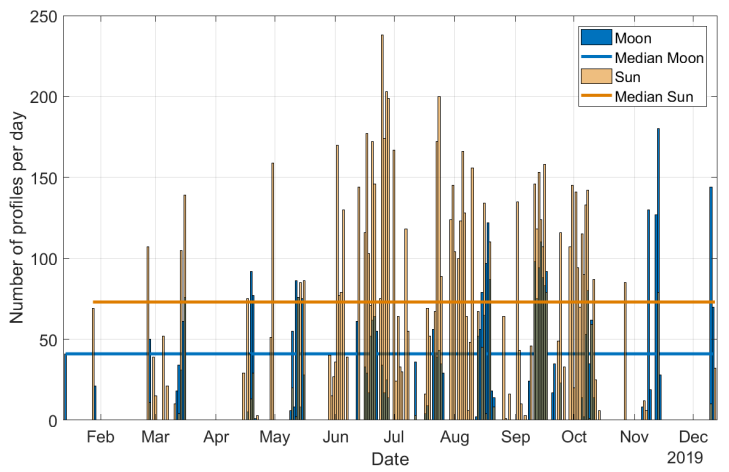

Figure 5. Number of $C_{n}^{2}(h)$ profiles per day over the year 2019

Fig.6 shows the probability density functions of all the layers probed by the PML for nightime (Moon) and daytime (Sun) conditions over the year 2019. We can see that PML is capable to reach an high precision and resolution in the site characterization. Several features of the $C_{n}^{2}(h)$ profiles can be clearly identified. For example, the Sun, by heating the ground, creates an active turbulent layer is the first hundreds of meters. This induces the first layers to be localized at median value of $1.2 \times 10^{-14} \mathrm{~m}^{-2 / 3}$ during the day while its PDF decreases at $2.9 \times 10^{-15} \mathrm{~m}^{-2 / 3}$ during the night and has a larger width. The PDFs of the layers between $5 \mathrm{~km}$ and $9.5 \mathrm{~km}$ are also very different between day and night. During daytime, the PDFs are much larger and spread over a large span of $C_{n}^{2}$ values $\left(10^{-16}-10^{-19} \mathrm{~m}^{-2 / 3}\right)$ while, during nighttime, the PDFs are much narrower and the range is much smaller $\left(3 \times 10^{-17}-3 \times 10^{-18} \mathrm{~m}^{-2 / 3}\right)$.
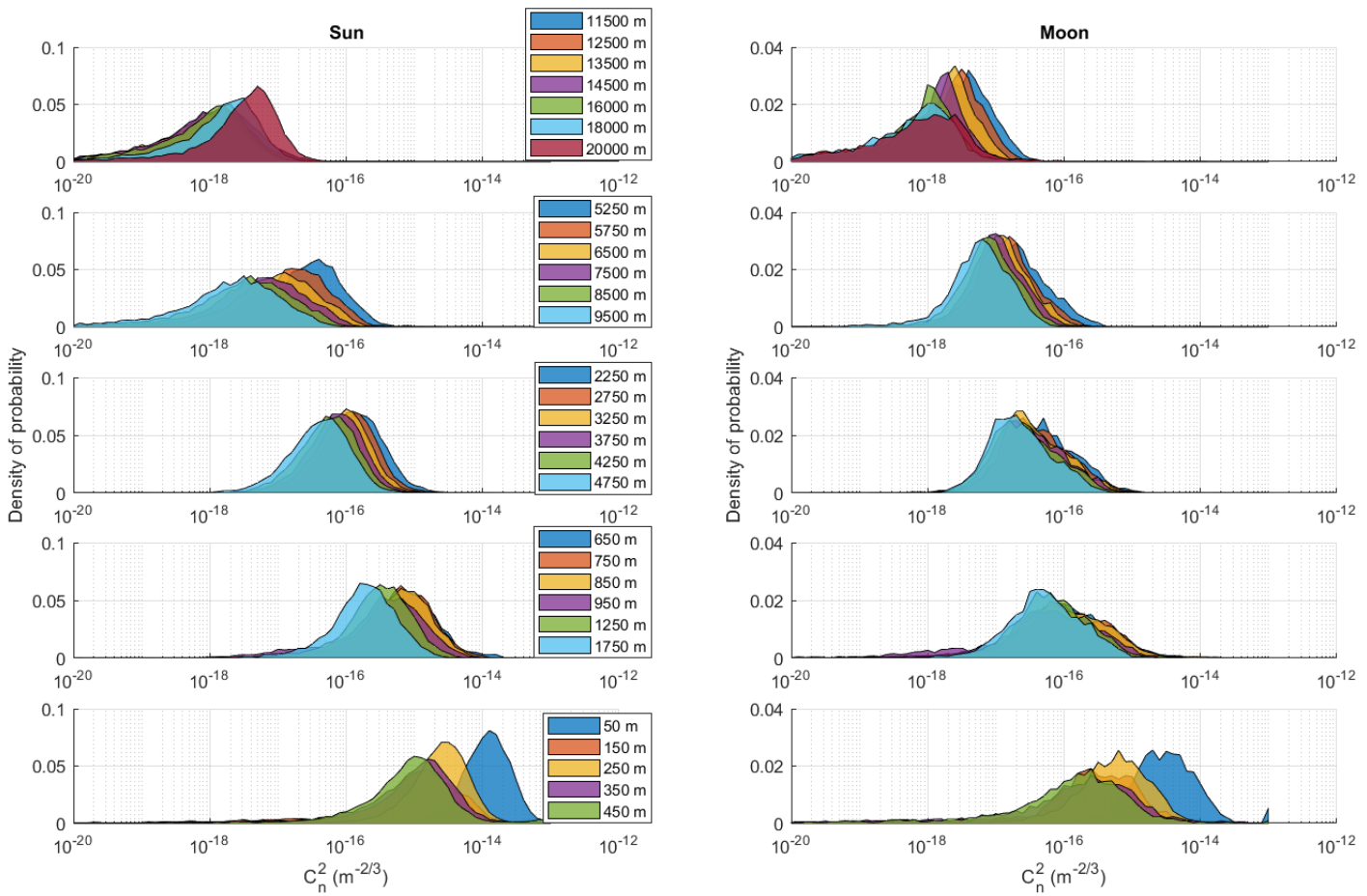

Figure 6. Probability density function of $C_{n}^{2}(h)$ for all the layers during day (Sun) and night (Moon) over the year 2019. 


\section{CONCLUSION}

This paper has presented the capabilities of PML for monitoring the atmpospheric turbulence at a given site: the Calern Observatory. The PML is a $C_{n}^{2}(h)$ profiler with high resolution in the atmosphere. This instrument is based on the measurement of the angular covariance of the AA along an extended object like the Moon or Sun. The observation statistics and some examples of the data acquired at Calern Observatory show that PML is a very good tool for turbulence monitoring and site testing. The instrument provides a detailed description of the $C_{n}^{2}(h)$ profile evolution over the year. This will help for the scheduling of the astronomical observations over the year and gives a precious input for turbulence forecasting models.

\section{ACKNOWLEDGMENTS}

A special thanks is addressed to technical team of the Lagrange Laboratory, particularly Christophe Bailet for his precious help and to the technical staff of the Calern observatory. We thank Flavien Blary and Mohammed Marjani for their contributions at the early stages of the project. The development of the PML instrument has been supported by the French National Institute for Universe Sciences in collaboration with the Observatoire de la Côte d'Azur and the University of Nice Sophia Antipolis with financial support from CNES and Federation Doblïn. This work was supported by the LABEX Cluster of Excellence FIRST-TF (ANR-10-LABX-48-01), within the Program "Investissements d'Avenir" operated by the French National Research Agency (ANR).

\section{REFERENCES}

1. Le Louarn, M., Hubin, N., Sarazin, M., and Tokovinin, A., "New challenges for adaptive optics: extremely large telescopes," Monthly Notices of the Royal Astronomical Society 317(3), 535-544 (2000).

2. Hubin, N., "Toward the adaptive optics for the $40 \mathrm{~m}$ class european elt.," in [In Second AO4ELT Conference], (2011).

3. Ellerbroek, B., "The tmt adaptive optics program.," in [In Second AO4ELT Conference.], (2011).

4. Costille, A. and Fusco, T., "Impact of the cn2 description on wide field AO performance," in [2nd Int. Conf. AO4ELT], (2011).

5. Carbillet, M., Aristidi, E., Giordano, C., and Vernin, J., "Anisoplanatic error evaluation and wide-field adaptive optics performance at dome c, antarctica," Monthly Notices of the Royal Astronomical Society 471(3), 3043-3050 (2017).

6. Robert, C., Conan, J.-M., and Wolf, P., "Impact of turbulence on high-precision ground-satellite frequency transfer with two-way coherent optical links," Phys. Rev. A 93, 033860 (Mar 2016).

7. Canuet, L., Védrenne, N., Conan, J.-M., Petit, C., Artaud, G., Rissons, A., and Lacan, J., "Statistical properties of single-mode fiber coupling of satellite-to-ground laser links partially corrected by adaptive optics," J. Opt. Soc. Am. A 35, 148-162 (Jan 2018).

8. Vedrenne, N., Velluet, M., Petit, C., Michau, V., Chabe, J., Ziad, A., Phung, D., Maurice, N., Samain, E., Artaud, G., Issler, J., Toyoshima, M., Akioka, M., Kolev, D., Munemasa, Y., Takenaka, H., and Iwakiri, N., "First results of wavefront sensing on sota," in [2015 IEEE International Conference on Space Optical Systems and Applications (ICSOS)], 1-8 (Oct 2015).

9. Horwath, J., Perlot, N., Knapek, M., and Moll, F., "Experimental verification of optical backhaul links for high-altitude platform networks: Atmospheric turbulence and downlink availability," International Journal of Satellite Communications and Networking 25(5), 501-528 (2007).

10. Giordano, C., Vernin, J., Vázquez Ramió, H., Muñoz-Tuñón, C., Varela, A. M., and Trinquet, H., "Atmospheric and seeing forecast: WRF model validation with in situ measurements at ORM," MNRAS 430, 3102-3111 (Apr. 2013).

11. Giordano, C., Vernin, J., Trinquet, H., and Muñoz-Tuñón, C., "Weather Research and Forecasting prevision model as a tool to search for the best sites for astronomy: application to La Palma, Canary Islands," MNRAS 440, 1964-1970 (May 2014).

12. Giordano, C., Rafalimanana, A., Ziad, A., Aristidi, E., J., C., Fante $\tilde{A}^{-}$-Caujole, Y., and Renaud, C., "Contribution of statistical site learning to improve optical turbulence forecasting," MNRAS (2020). 
13. Aristidi, E., Ziad, A., Chabé, J., Fantéi-Caujolle, Y., Renaud, C., and Giordano, C., "A generalized differential image motion monitor," Monthly Notices of the Royal Astronomical Society 486, 915-925 (04 2019).

14. Chabé, J., Aristidi, E., Ziad, A., Lantéri, H., Fanteї-Caujolle, Y., Giordano, C., Borgnino, J., Marjani, M., and Renaud, C., "Pml: a generalized monitor of atmospheric turbulence profile with high vertical resolution," Appl. Opt. 59, 7574-7584 (Sep 2020).

15. Ziad, A., Blary, F., Borgnino, J., Fanteï-Caujolle, Y., Aristidi, E., Martin, F., Lantéri, H., Douet, R., Bondoux, E., and Mékarnia, D., "First results of the pml monitor of atmospheric turbulence profile with high vertical resolution," $A \mathscr{S} A$ 559, L6 (2013).

16. Avila, R., Ziad, A., Borgnino, J., Martin, F., and Agabi, A., "Theoretical spatiotemporal analysis of angle of arrival induced by atmospheric turbulence as observed with the grating scale monitor experiment," $J$. Opt. Soc. Am. A 14, 3070-3082 (1997). 